\title{
Ultrasound as a New Imaging Tool to Assess Pathological Change of Joints in Preclinical Mouse Models of Osteoarthritis
}

\author{
Lin $\mathrm{X}$ and Xing $\mathrm{L}^{*}$
}

Department of Pathology and Laboratory Medicine, University of Rochester Medical Center, Rochester, USA

*Corresponding author: Xing L, Department of Pathology and Laboratory Medicine, University of Rochester Medical Center, Rochester, 601 Elmwood Ave, NY 14642, USA, Fax: 1-5857564468, Tel: 1-5852734090, E-mail: Lianping_xing@urmc.rochester.edu

Citation: Lin X, Xing L (2018) Ultrasound as a New Imaging Tool to Assess Pathological Change of Joints in Preclinical Mouse Models of Osteoarthritis. J Orthop Physiother 1(1): 102. doi: 10.15744/2639-930X.1.102

Received Date: December 30, 2017 Accepted Date: September 18, 2018 Published Date: September 20, 2018

\begin{abstract}
Murine osteoarthritis (OA) models are important for exploring OA pathology and treatment in the pre-clinical study. Longitudinal in vivo imaging modalities, including X-ray, computed tomography and magnetic resonance imaging, are commonly used diagnostic tools in OA clinic, while end-point histomorphometry analysis is the major outcome measurement in pre-clinical study because clinical imaging modalities have limited resolution for small animals. Thus, developing new longitudinal in vivo imaging protocols for murine models of $\mathrm{OA}$ is a critical unmet need. Here, commonly used post-traumatic murine models of OA and the utilization of X-ray, computed tomography and magnetic resonance imaging techniques to monitor disease progression and treatment response in these models is reviewed. Then ultrasound (US) imaging, a widely used and cost-efficient tool, in arthritis clinic and its utilization in knee OA of patients is introduced. Finally, our experience of using US imaging in normal and OA mouse knees is described to demonstrate the feasibility of US as a new imaging tool to measure disease progression longitudinally.
\end{abstract}

Keywords: Osteoarthritis; Murine Models; Post-Traumatic; in vivo Imaging; Ultrasound

\section{Osteoarthritis (OA)}

Osteoarthritis is the most common form of arthritis that affects millions of adults worldwide [1]. There are no effective drugs for $\mathrm{OA}$ and current treatments are mostly palliative. OA is a whole joint disease involving cartilage, subchondral bone and synovial soft tissue [2]. Many factors contribute to OA pathogenesis, including mechanical stress, biochemical abnormalities, and metabolic disorders [3]. Murine models are important for OA research for the ease of genetic manipulation and inflicting joint trauma by surgery [4]. A challenge for murine models of OA is the lack of non-invasive approaches that allow researchers to assess the severity and progression of OA diseases longitudinally. X-ray and magnetic resonance imaging (MRI) are widely used in OA patients with established quantitative and semi-quantitative assessment standards [5-8]. However, use of these imaging modalities in small animals, especially mice, is limited due to expensive and low resolution equipment, and operator dependence. Medical ultrasound (US) has also been used to evaluate knee OA disease activity in patients, demonstrating that US detected synovial inflammation and effusion is positively correlated with radiographic OA and clinical symptoms [9]. Our group applied US scan on joints of TNF-transgenic mice, a mouse model of rheumatoid arthritis (RA) and demonstrated that both joint space volume and power doppler (PD) volume can be used as outcome measures of joint inflammation and active synovitis [10,11]. We have started to use US scanning in mouse OA and obtained promising results [12]. Here, the advantages and limitations of existing imaging modalities in murine models of OA are reviewed. Our own experience using US imaging modality in mouse OA joints is described, and the possibility to use longitudinal US as a new approach to quantify joint soft tissue changes in a mouse model of OA is discussed.

\section{Murine models of OA}

Preclinical OA models have greatly improved our understanding of OA pathogenesis. Multiple animal species have been used, including canine, goat, pig, horse, and rhesus macaque [13-21]. Mouse OA models are the most commonly used preclinical OA models due to the ease of surgical or genetic manipulation, drug administration, and their relatively low cost. Murine models of $\mathrm{OA}$ are roughly categorized into the following groups: surgery-induced, genetic- and chemical-induced joint injury. 
Surgery-induced OA: This OA model mimics a pathologic process of posttraumatic OA (PTOA) in humans. It is achieved via joint destabilization caused by various surgical procedures in the knees. A mouse knee joint is composed of hard tissues and surrounding soft tissues that we named synovial soft tissues. Hard tissues consist of articular cartilage of the femur (lateral or medial condyle) and tibia (tibia plateau) and subchondral bones. Soft tissues include ligaments (medial and lateral collateral, anterior and posterior cruciate, patellar, medial meniscus tibial/coronary line), the meniscus (medial and lateral), and the synovial membrane. By transecting or one or several joint ligaments in combination of removing a portion of medial meniscus, mice develop osteoarthritic changes at different paces, depending on the procedure (Figure1). Among commonly used mouse PTOA models, the most severe cartilage damage is seen in knee that undergoes anterior cruciate ligament transaction (ACLT), followed by MLI (meniscal-ligamentous Injury)- transection of posterior cruciate ligament plus removal of medial meniscus and then by DMM (destabilization of the medial meniscus)-transection of the medial meniscus tibial ligament [22-25]. At the 2016 OARSI meeting, 133 out of over 900 abstracts used PTOA mouse models. Of these, 53 used the DMM model, 78 used the ACLT model and 2 used the MLI model. Surgical induced OA models ensure that OA is induced at the same time, thus minimizing the variation of the onset of $\mathrm{OA}$ in the genetic models. The combination of DMM and genetically modified animals are used to identify enzymes, proteins and transcriptional changes in OA pathogenesis [26-30]. In patients, PTOA is often diagnosed 15-20 years after the initial joint injury [31]. The DMM involves transecting the medial meniscal tibial ligament, which doesn't exist in patients. The ACLT often causes acute joint damage and severe inflammation and the MLI requires highly experienced personnel. Despite these limitations, surgery-induced mouse models of OA are still most commonly used in pre-clinical studies.

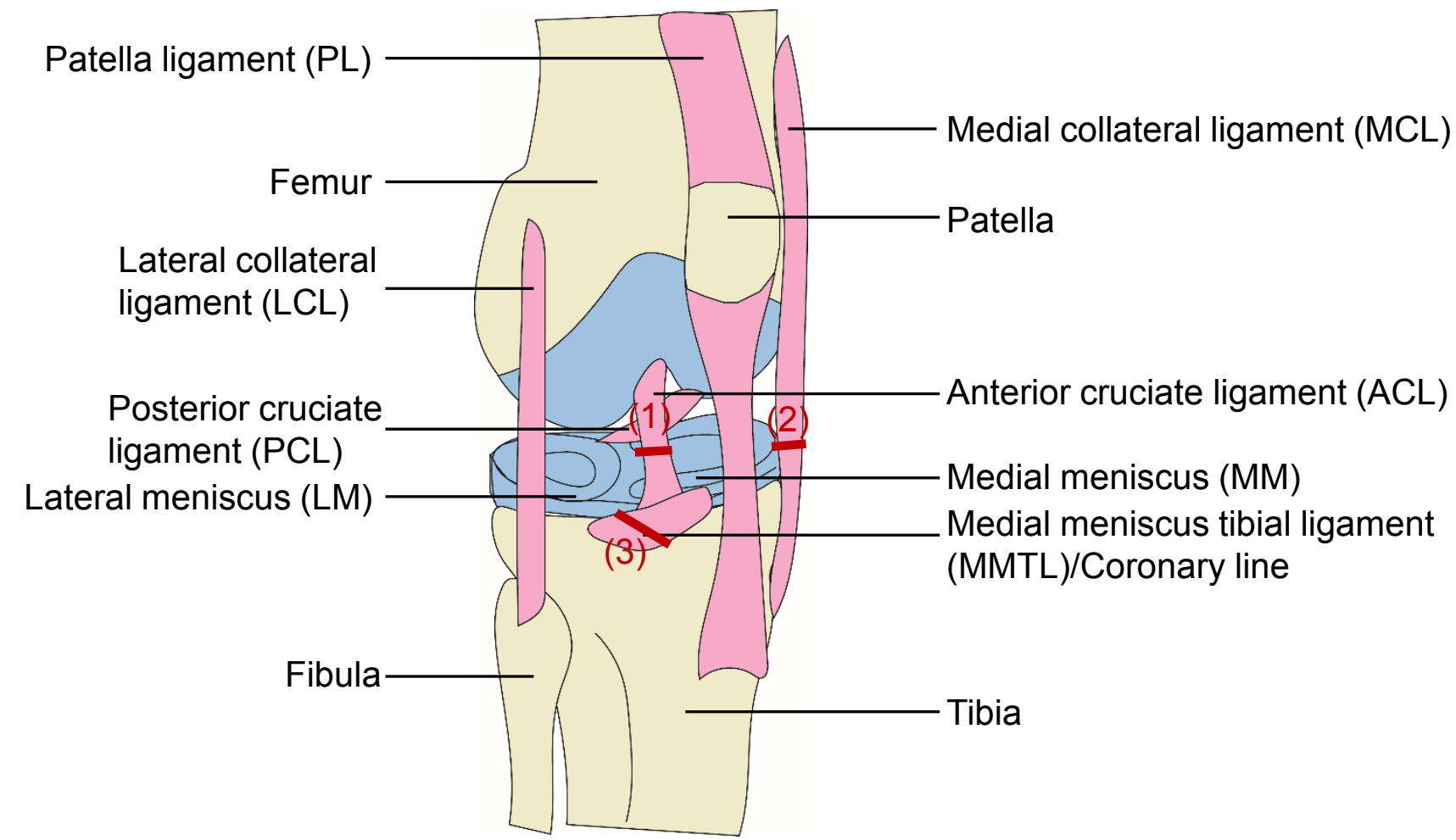

Figure 1: A mouse knee joint and surgery procedures that induce posttraumatic OA. Scheme of a mouse knee joint. Potential targets of posttraumatic OA model are indicated in numbers. (1) ACLT (anterior cruciate ligament transaction): transection of ACL. (2) MLI (meniscal-ligamentous Injury): transection of MCL plus removal of medial meniscus. (3) DMM (destabilization of the medial meniscus): transection of MMTL

Genetically modified mice with OA phenotypes: In different strains of wild-type mice, OA histological changes could occur spontaneously as mice aged, including C57Bl/6, BALB and Str/ort mice [32,36]. Although these spontaneously developed OA mice bear similar degenerative natures of human OA, it takes a long time to observe age-related OA changes. For instance, it takes 24-months for changes to become observable in C57Bl/6, 12-months in BALB mice, and 2.5-months in Str/ort mice $[32,36]$. Genetically modified mice show that many genes are involved in OA development. More than 18 different genetically different mouse strains were used in OA preclinical study as early as 1956 [37]. The ease of genetic engineering in mice has greatly advanced our understanding of OA pathology. The genetically engineered mice can be broadly classified into four broad categories dependent on gene or genes that are manipulated: cartilage matrix degradation, terminal (hypertrophic) chondrocyte differentiation or apoptosis, inflammation or synovitis, and bone turnover [38]. For example, a mutation in collagen or collagenase leads to the development of $\mathrm{OA}$ at a different age, confirming the crucial role of cartilage in OA development [39-41].

Non-surgical-induced OA: This is caused by intra-articular injection of chemicals or proteinases including iodoacetate, papain, or collagenase, which induces joint swelling and OA histological changes at greater rates of speed and severity [42-45]. Although non-surgical-induced OA has less variation among animals and fast development of OA-related pain in pre-clinical OA research, 
its pathogenesis does not really represent the development and progression of human OA. As a result, it is not the ideal option for OA pre-clinical study and often requires another model for validation.

Limitations of murine models of OA: Preclinical mouse OA models greatly advance our understanding of OA pathogenesis and provide a valuable tool to evaluate new OA drugs. However, a major limitation of preclinical models is that the anatomy and physiology between mice and human is not the same. For instance, human do not have the MMTL ligament. Moreover, unlike a variety of imaging modalities available to OA patients, preclinical moue OA models do not have an inexpensive and efficient imaging modality to monitor disease progression longitudinally. The primary outcome measurement of murine models of OA is structural damage or erosion of articular cartilage. The assessment of severity of OA relies on endpoint histology parameters such as the OARSI scoring and cartilage area [46]. Using endpoint assessment as primary outcome measurement greatly increases the number of experimental mice and associated cost. More importantly, the OA animal models vary in terms of severity and progression, thus evaluating OA progression with an in vivo imaging modality before the endpoint outcome measurement is beneficial. One of the most important symptoms of human OA, which can only be measured indirectly by parameters in mouse OA models.

\section{In vivo imaging methods for murine OA models}

Commonly used imaging modalities for human OA include X-ray, microCT ( $\mu$-CT) and magnetic resonance imaging (MRI) to observe changes in the anatomic feature, cartilage, subchondral bone, and synovium. These techniques have also been applied to murine OA models with limitations.

X-ray: Radiography is a commonly used tool in the diagnosis of OA in patients. In 1957, Kellgren and Lawrence established semi-quantitative assessments of knee OA on X-ray film. Known as the KL grading system, this uses 5 grades of osteophyte formation that include narrowness of joint space, subchondral bone sclerosis, and morphometry change of the articular surface [5]. Due to the heterogeneity of OA pathology, the occurrence of these X-ray-based biomarkers varies among individuals and grading of joint space narrowing and osteophyte is observer-dependent [47,48]. A quantitative assessment of joint space width to better characterize OA progression was developed around the 1990s and its sensitivity was validated with MRI imaging [49-51]. However, radiographic imaging is limited to the observation of bone tissue, cartilage loss, and deformation at a very late stage of the disease. Meniscus, synovitis, joint swelling that manifest in the early stages of the disease cannot be detected with radiography. Moreover, few studies utilize radiography on murine models of OA due to limited resolution of small animal X-ray [24]. Moreover, joint space narrowing in mice OA happens at end stage disease, where OARSI score is 5-6, whereas most OA studies are concerned with early stage disease in order to identify new therapeutic targets.

$\boldsymbol{\mu}$-CT: Subchondral bone remodeling is one of the characteristics of OA, including change in bone volume, growth plate morphology and increased bone turnover [52]. CT and high resolution CT is an X-ray based high-resolution imaging method optimal in evaluating subchondral bone plate morphology, thickness, trabecular pattern, osteophyte formation and calcification in the tendon $[53,54]$. Based on the utility of CT in clinic, laboratory $\mu$-CT was developed and first used in a spontaneous OA mice model in 2004 to observe osteophyte, trabecular remodeling, subchondral bone plate thickening and sclerosis [55]. An ex vivo quantitative $\mu$-CT method was later developed to evaluate the subchondral bone change in a collagenase-induced OA mice model [56]. McErlain et al. used in vivo $\mu$-CT to measure volumetric bone mineral density in the subchondral area in rats with $\mathrm{OA}$ and found that they had lower bone mineral density four months after ACLT [57]. Botter et al. used in vivo $\mu$-CT to evaluate cartilage damage and osteophytosis in a chemical-induced mouse OA model and found more cartilage loss, more osteophytosis, growth plate thinning, and increase in growth plate porosity at an early stage of OA, which could not be seen later [58]. In addition to assessing subchondral bone, $\mu$-CT can also assess articular cartilage with phase-contrast imaging. Kotwal et al. used ionic contrast reagent Ioxaglate to detect the decrease of cartilage thickness in exercise-induced OA in mice ex vivo and validated their $\mu$-CT findings with histological and biochemical methods [59]. Ruan et al. used contrast reagent osmium tetroxide for ex vivo contrast-enhanced $\mu$-CT scanning in cruciate ligament injury and a DMM OA mouse model. They did 3D reconstruction to quantify cartilage volume, cartilage surface and bone surface area and validated results with histological scoring [60]. Lakin et al. further analyzed tibial cartilage glycosaminoglycan content in a collagenase-induced OA mice model ex vivo. They showed that glycosaminoglycan content measured with contrast-enhanced $\mu$-CT correlated well with Safranin staining, validating their $\mu$-CT findings [61]. It should be noted that although some ex vivo $\mu$-CT imaging modality yields high resolution cartilage data, it is more similar to endpoint measurements such as histomorphometric analysis. This data should not be compared to data acquired from in vivo imaging. Moreover, since $\mu$-CT is an X-ray based imaging modality it is not optimal for evaluating changes in soft tissues.

MRI: MRI is ideal for arthritic imaging due to its ability to distinguish the following joint soft tissues: cartilage, subchondral sclerosis, synovial fluid, osteophyte, ligament, patella position, and synovitis. Semi-quantitative standard, Whole-Organ Magnetic Resonance Imaging Scoring (WORMS) has been established for human knee OA, taking cartilage, marrow abnormality, bone cysts, bone attrition, osteophytes, compartment, menisci, ligaments and synovitis into consideration [7]. Ostergaard et al. found that synovial volume measured with MRI is highly correlated with the synovial hypertrophy scoring in RA patients, which is an indicator of inflammation of arthritis [62]. Our group used MRI to observe the progression of joint tissue damage in an RA mouse 
model and developed quantitative MRI-based biomarkers. These biomarkers include synovial volume and popliteal lymph node volume validated with $\mu \mathrm{CT}$ and histology analysis $[63,64]$. MRI is also applied to small animal OA models to evaluate cartilage and soft tissues. Munasinghe et al. used MRI to monitor joint structures of exercise-induced OA in 3-, 7-, and 12-month old Str/ Ort mice. They found thickening of the patellar tendon, deformity, and sclerosis in 7-12 month-old OA mice, which was more severe in males than in females $[65,66]$. Goebel et al. assessed changes of femorotibial cartilage in rat knees that received ACL or sham surgery by 3D MRI at days 8, 14, 21, 40 and 60 post-surgery. Results indicated that mean cartilage thicknesses in OA knees decreased at an early phase (day 8, day 14) compared to sham knees and remained relatively stable thereafter. The histological correlation was significant only in untouched healthy cartilages [67]. These studies demonstrate the ability of MRI technique in assessing OA-related changes in femorotibial cartilage volume/thickness. Although MRI has been established as a useful tool to measure changes in soft tissues (i.e. synovial volume) it is very expensive. MRI data processing is time-consuming and requires specially trained operators. Seeking another imaging modality for mouse OA study is therefore encouraged.

Single photon emission computed tomography (SPECT) and positron emission tomography (PET): Scintigraphy is an imaging module in which radioisotopes are attached to drugs that travel to a specific organ or tissue (radiopharmaceuticals). The drugs are injected systemically and the emitted radiation is captured by external detectors (gamma cameras) to form $2 \mathrm{D}$ images. It is widely

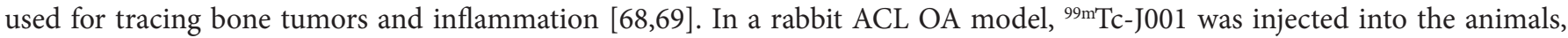
internalized by macrophages and detected by scintigraphy, which showed that OA knee joint has higher scintigraphic signals. This showed higher internalization of ${ }^{99 \mathrm{~m}} \mathrm{Tc}$-J001, suggesting higher bone metabolism in OA [70]. SPECT also uses gamma cameras to capture emitted radiation from radioisotopes to generate 3D images [71]. SPECT was used with $\mu$-CT to monitor bone turnover in a MLI-induced OA model in rats. While $\mu$-CT was used for anatomic localization, SPECT showed increased subchondral turnover in OA joints when compared to healthy joints [53]. Similarly, radiolabeled tracer such as ${ }^{18} \mathrm{~F}-$ fluoride and ${ }^{18} \mathrm{~F}-\mathrm{FDG}$, are used to assess metabolism in PET imaging. Franc et al showed that both acute and chronic phases of arthritis have higher PET signal with ${ }^{18} \mathrm{~F}$-AraG tracer in a mice adjuvant arthritis mouse model [72]. However, radioisotope methods had a resolution of 1-2mm, which is insufficient to depict the anatomy of mice knee joint [47].

\section{Ultrasound (US) as a new imaging tool for synovial changes in OA}

US is to send pulses of US into tissue and then record sound echoes from the tissue as B-mode images or color Power Doppler (PD) signals, both of which can be reconstructed into a 3D volume measurement. Different tissue types have different echogenic properties, depending on their location relative to the surface, density, and fluid content [73]. B-mode US image arises from the coherent interaction of random scatterers within a resolution cell when a certain anatomical region is scanned [73]. Twodimensional images of the tissue are acquired with B-mode imaging, allowing researchers to inspect the anatomy of the tissue and navigate the identification of the region of interest for Power Doppler imaging. The Doppler Effect is named after Austrian physicist Christian Doppler in 1842. It describes the change in frequency of a wave (or another periodic event) for an observer moving relative to its source. Doppler ultrasound permits real-time viewing of blood flow through a blood vessel, and this method has been used to evaluate the major arteries and veins of the body, the heart, and in obstetrics for fetal monitoring. Power Doppler is one of Doppler ultrasound techniques with high sensitivity that could also detect the direction of flow.

Rheumatologists started using US imaging to evaluate joint and soft tissue in the 1990s [74,75]. Since then, utilization of US in RA clinic has been shown to be beneficial to RA diagnosis [76-79]. The 2010 new classification criteria for RA issued by American College of Rheumatology (ACR) and European League against Rheumatism (EULAR) focused more on inflammatory changes in the joint, which is optimal for US imaging. In 2013, EULAR issued ten recommendations for RA imaging in clinical management, nine that involve US, which popularized its use in in RA clinics [80-83].

US imaging in human OA joints: OA pathology includes inflammatory changes in the joint, providing the rationale to use US to monitor OA progression and its responsiveness to therapy. In fact, the utilization of US imaging grows rapidly in OA clinics because structural abnormalities detected by the US are commonly correlated with clinical endpoints [84-87]. US are more sensitive in detecting osteophytes and cartilage changes during arthroscopy [88-90]. To avoid confusion with terminology used in traditional radiology and MRI findings, the term "ultrasonographic" is added prior to the observed physical or pathological anatomy structures. US-based biomarkers for OA have been developed according to findings in several clinical studies [9,91-95]. For instance, synovial thickness $>=4 \mathrm{~mm}$ is referred to as ultrasonographic synovitis in OA in an EULAR clinical study. This is validated with X-ray [91,92]. Suprapatellar or synovial effusion depth $>=4 \mathrm{~mm}$ is diagnosed as ultrasonographic effusion [9,93]. In 2016, the reliability of US biomarkers on US findings from 13 patients with early knee OA was evaluated by 11 US experts in the Outcome Measures in Rheumatology (OMERACT) US Task Force on knee OA. A semi-quantitative scoring system was used to measure ultrasonographic synovitis, osteophytes, cartilage and meniscal damage. The results moderate to good intra- and interobserver reliability scores for synovitis and fair to good of intra- and inter-observer reliability scores for cartilage or meniscal damage and osteophytes range, respectively. This suggests that using a standardized protocol and semi-quantitative US scoring of pathological changes in knee OA can be reliable [96].

US imaging system for healthy mouse knee: To determine whether US can be used to evaluate pathology of knee joints in mice, 
we examined a WT mouse knee with small animal US system. Although various small animal ultrasound systems are commercially available, we used Vevo770 (VisualSonics, Toronto, Canada) because the RMV704 transducer had a center frequency of $40 \mathrm{MHz}$, with an axial resolution of $40 \mu \mathrm{m}$ and a lateral resolution of $70 \mu \mathrm{m}$, which is most appropriate for the observation of mouse knee joint (Table 1). The highest center frequency of the L38-22v CMUT transducer from VeraSonics is $28 \mathrm{MHz}$, which is not sensitive enough for the observation of mouse knee joint. On the other hand, the center frequency of ultrasound biomicroscope systems could be as high as $275 \mathrm{MHz}$, allowing the observation at a cellular level, which is also not appropriate for joint imaging. In brief, anesthetized mice are placed in a supine position with their knees flexed over a customized mould to an approximately $135^{\circ}$ angle. US gel is applied between the skin and the 704 US scan head. The knee joint is scanned in a vertical direction. US detection presents as a triangular area underneath of skin, which can be delineated by a hyperechoic line of femoral and tibial articular cartilage (Figure 2). Histology of the same region revealed that it is composed of the patellar ligament, soft tissues including synovium, fat pad, soft tissue portion of the meniscus, and empty (synovial) space (Figure 3A). Histology observation cannot be precisely matched to US findings due to the following reasons: 1) US could detect fluid in the synovial space (Figure 3B). However, histological analysis requires fixation, dehydration, and sectioning, which results in the loss of joint fluid. 2) US wave is completely reflected at calcified bone surface. Anything beneath calcified bones is not detected in US images. Thus if calcification occurs in the synovium or the meniscus, anything beneath calcification is not detected. 3) US detection reflects the 3D shape of joint soft tissues, while histology only reflects a $2 \mathrm{D}$ image of a joint at certain embedded rotation. Thus, ultrasound findings in the knee joint is defined as US synovial volume (USSV), which includes the space below patellar ligament and above mature bone tissue of the femur, tibia, and meniscus. This differs from synovial space in an H\&E-stained section in which normal knee joints only contain the space between the meniscus and articular cartilage, which is very small. However, USSV is larger in a normal joint that contains various soft tissues and fluid.

\begin{tabular}{|c|c|c|c|c|}
\hline Manufacturer & Transducer & $\begin{array}{c}\text { Center frequency } \\
(\mathrm{MHz})\end{array}$ & $\begin{array}{l}\text { Axial resolution } \\
\qquad(\mu \mathrm{m})\end{array}$ & $\begin{array}{l}\text { Lateral resolution } \\
\qquad(\mu \mathrm{m})\end{array}$ \\
\hline Verasonics & L38-22v CMUT & 28 & - & - \\
\hline Visualsonics Vevo3100 & RMV704 & 60 & 40 & 70 \\
\hline Visualsonics Vevo3100 & MX700 & 70 & 30 & 65 \\
\hline Ultrasound Biomicroscopes ${ }^{1}$ & LN_300 & 275 & 6.2 & 6.4 \\
\hline
\end{tabular}

Table 1: Comparison of different ultrasound system

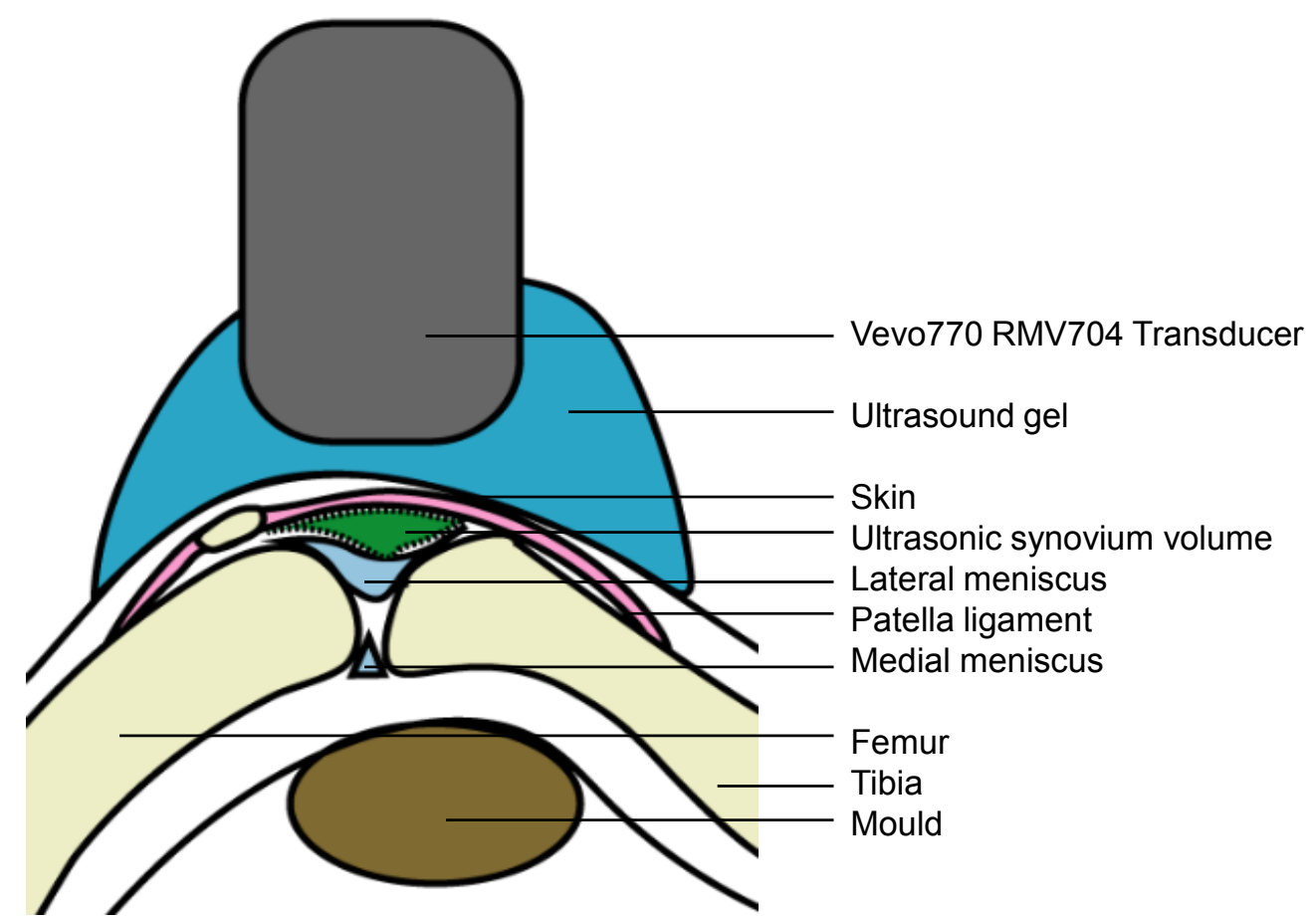

Figure 2: Ultrasound scanning of a mouse knee joint. Knee joint is bent at an angle with a mould underneath. Centrifuged ultrasound gel is sprayed on the skin surface after completely removing fur with depilatory cream. The Vevo ultrasound RMV704 transducer is lowered and immersed in the gel to acquire 2D B-mode ultrasound images. For 3D data acquirement, the scan head moves in a set range vertical to the paper 

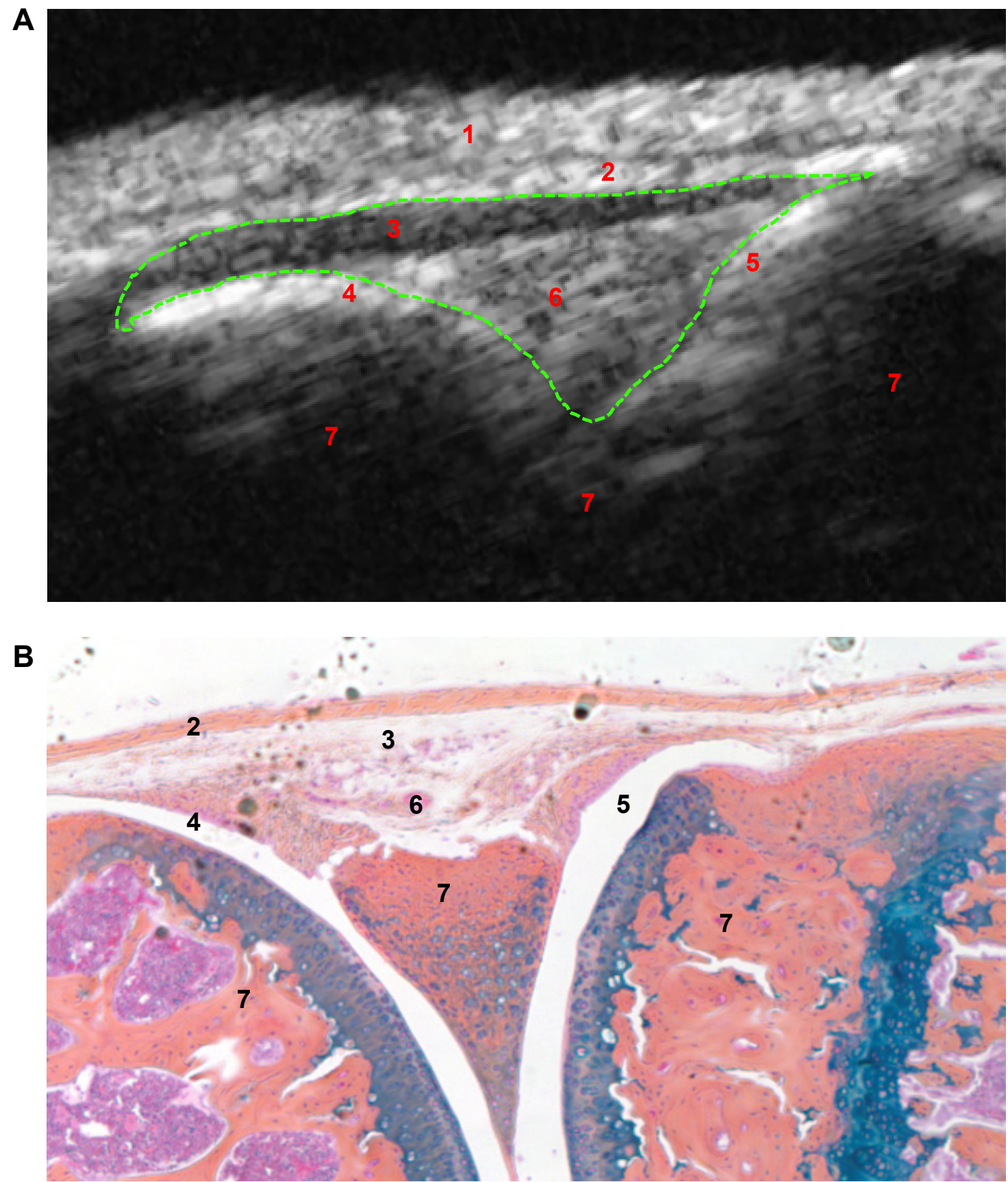

Figure 3: Ultrasound imaging of $\mathrm{C} 57 \mathrm{Bl} / 6$ male mouse knee at 3 month of age. (A) B-mode US imaging of the knee joint. The segmentation of US synovial volume is outlined by green USSV. Numbers indicate structures corresponding to those in histology. 1: Skin and hair. 2: Patellar ligament. 3: Fat pad. 4\&5: Synovial space. 6: Connective tissue. 7:Calcified bone tissue. (B) Alcian blue/Orange G staining of the same knee joint

US imaging in mouse RA knee: Dr. Schwarz's group used US to examine joints of TNF-Tg mice, a mouse model of RA (11), because US is frequently used in RA clinic due to its nature of severe inflammation. They detected significantly increased USSV in TNF-Tg mouse joints, which correlates strongly with synovial volume measured with CE-MRI (63). Furthermore, the RA joints also have increased PD volume (PDV) measured with PD-US, which represents the volume of blood vessels. The PD signal is further validated by immunohistochemistry staining showing CD31 positive staining at the same site.

US in mouse OA joints: Compared to RA, OA joints have a much lower degree of inflammation and perhaps other soft tissue changes, making US detection more difficult. Spriet et al. first observed femoral cartilage damage in a rabbit ACLT OA model in vitro with B-mode US biomicroscopy. They semiquantitatively graded their US finding and validated US grade with histology grade, proving the potential of using US to monitor OA progression in small animals [97]. However, translating US biomicroscopy technique requires opening joint capsule to expose joint surface, which is invasive and cannot be used longitudinally.

In vivo US imaging protocol in an MLI OA mouse model (Figure 4). B-mode US at 4 and 11 weeks post-MLI on the same joints was performed. At the 4 week point, USSV was $2.76+/-0.54\left(\mathrm{~mm}^{3}\right)$ in MLI joints compared to $1.96+/-0.26\left(\mathrm{~mm}^{3}\right)$ in sham joints 
( $\mathrm{p}=0.005$, un-paired t-test). At the 11 week point, USSV increased to $4.7+/-0.64\left(\mathrm{~mm}^{3}\right)$ in MLI joints while USSV remained unchanged $\left(1.92+/-0.24 \mathrm{~mm}^{3}\right)$ in sham joints $(\mathrm{p}=2.43 \mathrm{E}-07$, un-paired t-test). More importantly, USSV was markedly increased at the same joint that received MLI ( $\mathrm{p}=6.66 \mathrm{E}-06$, paired $\mathrm{t}$-test), but not in joints that received sham operation ( $\mathrm{p}=0.84$, paired t-test). A significant change in MLI joints (73.14 +/- 17.56\% in MLI vs. $0.07+/-18.06 \%$ in sham, $p=1.8 \mathrm{E}-04$, un-paired t-test) was detected when the percentage change of USSV between 4 and 11 weeks was calculated. An average of USSV in normal knee joints of 30 mice is $1.97+/-0.17\left(\mathrm{~mm}^{3}\right)$. Thus, a standard normal USSV in adult mice is below $2.15 \mathrm{~mm}^{3}$. Increased PDV was found in mouse knees that received Hulth-Telhag surgical procedure that combines MLI and ACLT, causing severe cartilage loss [12,98].

A
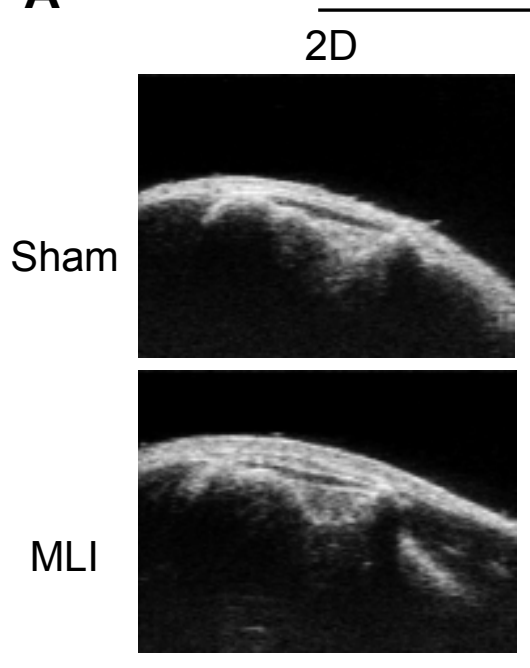

4 wks

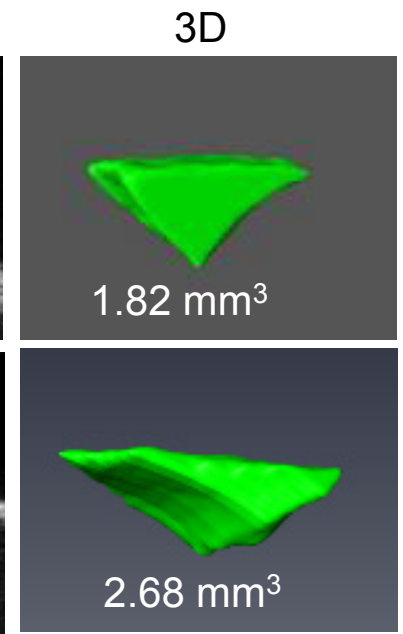

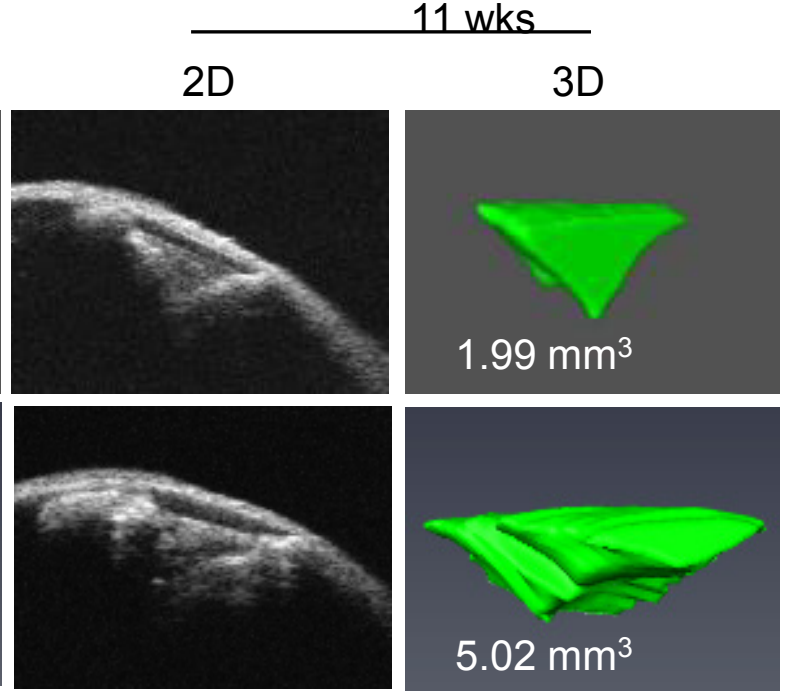

\section{B USSV $\left(\mathrm{mm}^{3}\right)$}

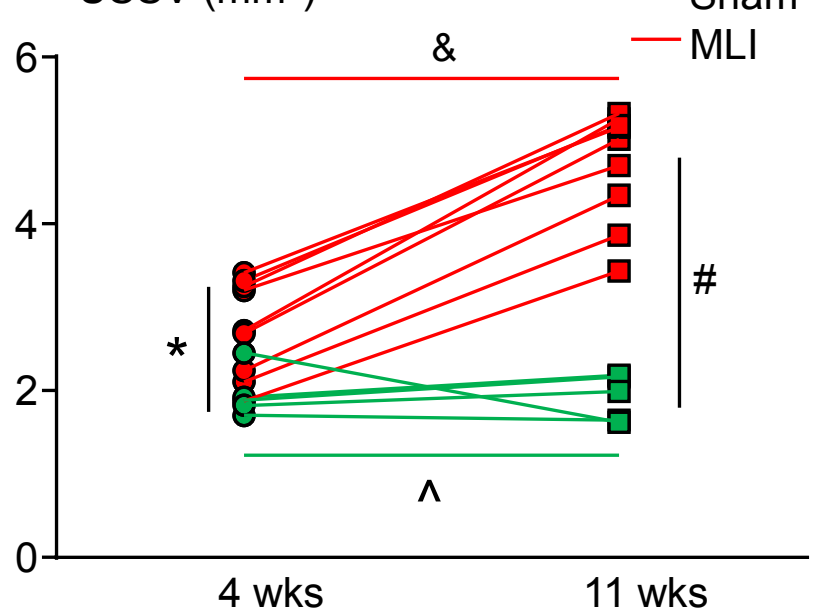

- Sham

\author{
* $p<0.05, M L I$ vs. sham at 4 wks \\ \# $p<0.05$, MLI vs. sham at $11 \mathrm{wks}$ \\ \& $p<0.05,4$ wks vs. 11 wks in MLI
}

$\wedge$
* \#: un-paired $t$ test

$\&, \wedge$ : paired $t$ test

Figure 4: Ultrasound imaging detects OA progression. KWT mice (male, 10-week-old) received MLI surgery on the right knee (N=9) and sham surgery on the left knee $(n=5)$. Mice were subjected to B-mode ultrasound scanning followed by $3 \mathrm{D}$ reconstruction and USSV measurement with Amira four and eleven weeks after MLI. (A) Representative ultrasound images of sham (green) and MLI (red) surgery mice 11 weeks after MLI.

(B) USSV increased in MLI but not sham surgery group

Based on these preliminary findings, US could be used to monitor OA disease progression in mouse PTOA models. It can detect early soft tissue changes prior to cartilage loss and allow tissue changes at the same joint to be compared [22]. Thus, quantitative USSV by US in OA joints have the following advantages 1) providing a 3D volumetric measurement of peri-articular soft tissue in real time; 2) randomizing experimental groups in intervention studies; 3 ) reducing the number of mice since the changes of USSV can be compared before and after the treatment at the same joint, and 4) enabling researchers to adjust the duration of treatment according to longitudinal USSV.

\section{Future perceptive}

By using the Vevo770 system with a US frequency of $40 \mathrm{MHz}$, our group has gained experience in US mouse knee joint and peri-articular soft tissues. However, US in mouse OA models also has its limitations. First, OA is a chronic disease with much less inflammation than RA. US may not be able to detect mild inflammatory changes and blood flow/vessels in peri-articular soft tissues of an OA joints. Second, the most common form of OA is an aging-related degenerative disease, which differs from 
PTOA used in our study. Soft tissue inflammation occurs in age related OA in mice has not been well studied, partially due to the lack of a non-invasive longitudinal imaging modality. Third, US imaging of knee joint cannot distinguish fine tissue structures detected by histology and cannot be used to replace histological analysis. Finally, imaging and data analysis vary among individual researchers who are sectioning the region of interest from each image frame and orientation of the knee joint. As a result, one single researcher has to complete all the US imaging sections and data analysis for a given experiment. To reduce the variation and increase scientific rigor, the US imaging and sectioning of the joint space volume should be standardized by recognition of anatomic landmarks in the knee joint, and US data should be analyzed blindly (and preferably) by a different researcher.

Some of these limitations are due to intrinsic nature of OA mouse models, and others may be related to the resolution of the Vevo770 system. The latest US machines such as Vevo3100 imaging system has better resolution The US frequency in the Vevo3100 imaging system is increased by 1.75 fold ( $70 \mathrm{MHz}$ vs. $40 \mathrm{MHz}$ in the Vevo770 system), greatly increasing the image resolution and enabling recognition of individual ligaments and meniscus. Another way to improve resolution is to use US-guided approach by injecting US contrast reagent into the knee joint. Furthermore, with enhanced resolution in the new imaging machine, we might be able to detect blood flow change in OA synovial soft tissues by PD-US.

Photoacoustic US has seen rapid growth as a biomedical imaging technique. In this imaging modality, endogenous energy is absorbed by the tissue and results in thermoelastic expansion of the tissue, which can be detected by an US transducer. Tissues with different blood flow and oxygenation status absorb heat differently, thus photoacoustic has been used in tumor diagnosis and other diseases [99]. Mice OA joints have thickened synovium and increased blood flow due to chronic inflammation, thus photoacoustic ultrasound shows great potential to monitor inflammation when combined with B-mode and Doppler ultrasound. However, it should be cautioned that since a healthy knee joint is not rich in blood vessels or soft tissues, it might be challenging to establish a basal level of photoacoustic signal. Moreover, typical OA develop milder synovitis than RA, thus it is more applicable to establish a robust photoacoustic protocol in the latter to determine whether this technique can detect small changes in blood flow and oxygenation status.

\section{Conclusion}

US imaging represents a promising alternative for OA imaging, especially in terms of observing synovial soft tissues during disease progression. Advantages of US imaging include no radioactive exposure, relatively low cost, easy to learn and less time-consuming when compared to MRI. With advancing imaging techniques, it is likely that in vivo US imaging may achieve higher resolution and render more information from a live mouse knee joint.

\section{Acknowledgement}

This work was supported by grants from the National Institute of Health, USA (AR63650 and AR069789) and NYSTEM, USA (CO-29548) to Lianping Xing.

\section{Compliance with ethics guidelines}

$\mathrm{Xi}$ Lin declares that she has no conflict of interest. Lianping Xing declares that she has no conflict of interest. All the animals used in this study were approved by Animal Care and Use Committee of University of Rochester, USA. This article does not contain any studies with subjects.

\section{References}

1. Lawrence RC, Felson DT, Helmick CG, Arnold LM, Choi H, et al. (2008) Estimates of the prevalence of arthritis and other rheumatic conditions in the United States. Part II. Arthritis Rheum 58: 26-35.

2. Goldring MB, Otero M (2011) Inflammation in osteoarthritis. Curr Opin Rheumatol 23: 471-8.

3. Samuels J, Krasnokutsky S, Abramson SB (2008) Osteoarthritis: a tale of three tissues. Bull NYU Hosp Jt Dis 66: 244-50.

4. Fang H, Beier F (2014) Mouse models of osteoarthritis: modelling risk factors and assessing outcomes. Nat Rev Rheumatol 10: 413-21.

5. Kellgren JH, Lawrence JS (1957) Radiological assessment of osteo-arthrosis. Ann Rheum Dis 16: 494-502.

6. Altman RD, Gold GE (2007) Atlas of individual radiographic features in osteoarthritis, revised. Osteoarthritis Cartilage 15: A1-56.

7. Peterfy CG, Guermazi A, Zaim S, Tirman PF, Miaux Y, et al. (2004) Whole-Organ Magnetic Resonance Imaging Score (WORMS) of the knee in osteoarthritis. Osteoarthritis Cartilage 12: 177-90.

8. Alizai H, Roemer FW, Hayashi D, Crema MD, Felson DT, et al. (2015) An update on risk factors for cartilage loss in knee osteoarthritis assessed using MRI-based semiquantitative grading methods. Eur Radiol 25: 883-93.

9. Conaghan PG, D’Agostino MA, Le Bars M, Baron G, Schmidely N, et al. (2010) Clinical and ultrasonographic predictors of joint replacement for knee osteoarthritis: results from a large, 3-year, prospective EULAR study. Ann Rheum Dis 69: 644-7.

10. Keffer J, Probert L, Cazlaris H, Georgopoulos S, Kaslaris E, et al. (1991) Transgenic mice expressing human tumour necrosis factor: a predictive genetic model of arthritis. EMBO J 10: 4025-31.

11. Bouta EM, Banik PD, Wood RW, Rahimi H, Ritchlin CT, et al. (2015) Validation of power Doppler versus contrast-enhanced magnetic resonance imaging quantification of joint inflammation in murine inflammatory arthritis. J Bone Miner Res 30: 690-4. 
12. Xu H, Bouta EM, Wood RW, Schwarz EM, Wang, et al. (2017) Utilization of longitudinal ultrasound to quantify joint soft tissue changes in a mouse model of posttraumatic osteoarthritis. Bone Res 5: 17012.

13. Pond, MJ, Nuki G (1973) Experimentally-induced osteoarthritis in the dog. Ann Rheum Dis 32: 387-8.

14. Little CB, Smith MM, Cake MA, Read RA, Murphy MJ, et al. (2010) The OARSI histopathology initiative - recommendations for histological assessments of osteoarthritis in sheep and goats. Osteoarthritis Cartilage 3: S80-92.

15. Cruz R, Ramírez C, Rojas OI, Casas-Mejía O, Kouri JB, et al. (2015) Menisectomized miniature Vietnamese pigs develop articular cartilage pathology resembling osteoarthritis. Pathol Res Pract 211: 829-38.

16. De Lasalle J, Alexander K, Olive J, Laverty S (2016) Comparisons among Radiography, Ultrasonography and Computed Tomography for Ex Vivo Characterization of Stifle Osteoarthritis in the Horse. Vet Radiol Ultrasound 57: 489-501.

17. Kamm JL, Frisbie DD, McIlwraith CW, Orr KE (2013) Gene biomarkers in peripheral white blood cells of horses with experimentally induced osteoarthritis. Am J Vet Res 74: 115-21.

18. Grynpas MD, Huckell CB, Reichs KJ, Derousseau CJ, Greenwood C, et al. (1993) Effect of age and osteoarthritis on bone mineral in rhesus monkey vertebrae. J Bone Miner Res 8: 909-17.

19. Duncan AE, Colman RJ, Kramer PA (2011) Longitudinal study of radiographic spinal osteoarthritis in a macaque model. J Orthop Res 29: 1152-60.

20. Uchihashi M, Hampel JA, Nemzek JA, Saccone PA, Eaton KA, et al. (2015) Use of Femoral Head and Neck Ostectomy and Physical Therapy to Manage Osteoarthritis in a Rhesus Macaque (Macaca mulatta). Comp Med 65: 260-5.

21. Ogawa S, Awaga Y, Takashima M, Hama A, Matsuda A, et al. (2016) Knee osteoarthritis pain following medial meniscectomy in the nonhuman primate. Osteoarthritis Cartilage 24: 1190-9.

22. Sampson ER, Beck CA, Ketz J, Canary KL, Hilton MJ, et al. (2011) Establishment of an index with increased sensitivity for assessing murine arthritis. J Orthop Res 29: 1145-51.

23. Hamada D, Sampson ER, Maynard RD, Zuscik MJ (2014) Surgical induction of posttraumatic osteoarthritis in the mouse. Methods Mol Biol 1130: 61-72.

24. Kamekura S, Hoshi K, Shimoaka T, Chung U, Chikuda H, et al. (2005) Osteoarthritis development in novel experimental mouse models induced by knee joint instability. Osteoarthritis Cartilage 13: 632-41.

25. Hayami T, Pickarski M, Zhuo Y, Wesolowski GA, Rodan GA, et al. (2006) Characterization of articular cartilage and subchondral bone changes in the rat anterior cruciate ligament transection and meniscectomized models of osteoarthritis. Bone 38: 234-43.

26. Glasson SS, Askew R, Sheppard B, Carito BA, Blanchet T, et al. (2004) Characterization of and osteoarthritis susceptibility in ADAMTS-4-knockout mice. Arthritis Rheum 50: 2547-58.

27. Glasson SS, Askew R, Sheppard B, Carito B, Blanchet T, et al. (2005) Deletion of active ADAMTS5 prevents cartilage degradation in a murine model of osteoarthritis. Nature 434: 644-8.

28. Glasson SS, Blanchet TJ, Morris EA (2007) The surgical destabilization of the medial meniscus (DMM) model of osteoarthritis in the 129/SvEv mouse. Osteoarthritis Cartilage 15: 1061-9.

29. Welch ID, Cowan MF, Beier F, Underhill TM (2009) The retinoic acid binding protein CRABP2 is increased in murine models of degenerative joint disease. Arthritis Res Ther 11: R14.

30. Loeser RF, Olex AL, McNulty MA, Carlson CS, Callahan M, et al. (2013) Disease progression and phasic changes in gene expression in a mouse model of osteoarthritis. PLoS One 8: e54633.

31. Lohmander LS, Englund PM, Dahl LL, Roos EM (2007) The long-term consequence of anterior cruciate ligament and meniscus injuries: osteoarthritis. Am J Sports Med 35: 1756-69.

32. Stoop R, van der Kraan PM, Buma P, Hollander AP, Billinghurst RC, et al. (1999) Type II collagen degradation in spontaneous osteoarthritis in C57Bl/6 and BALB/c mice. Arthritis Rheum 42: 2381-9.

33. Lapveteläinen T1, Hyttinen MM, Säämänen AM, Långsjö T, Sahlman J, et al. (2002) Lifelong voluntary joint loading increases osteoarthritis in mice housing a deletion mutation in type II procollagen gene, and slightly also in non-transgenic mice. Ann Rheum Dis 61: 810-7.

34. Lapveteläinen T, Nevalainen T, Parkkinen JJ, Arokoski J, Kiraly K, et al. (1995) Lifelong moderate running training increases the incidence and severity of osteoarthritis in the knee joint of C57BL mice. Anat Rec 242: 159-65.

35. Poulet B, Westerhof TA, Hamilton RW, Shefelbine SJ, Pitsillides AA (2013) Spontaneous osteoarthritis in Str/ort mice is unlikely due to greater vulnerability to mechanical trauma. Osteoarthritis Cartilage 21: 756-63.

36. Mason RM, Chambers MG, Flannelly J, Gaffen JD, Dudhia J, et al. (2001) The STR/ort mouse and its use as a model of osteoarthritis. Osteoarthritis Cartilage 9: 85-91.

37. Jay GE Jr, Sokoloff L (1956) Natural history of degenerative joint disease in small laboratory animals. II. Epiphyseal maturation and osteoarthritis of the knee of mice of inbred strains. AMA Arch Pathol 62: 129-35.

38. Little CB, Hunter DJ (2013) Post-traumatic osteoarthritis: from mouse models to clinical trials. Nat Rev Rheumatol 9: 485-97.

39. Säämänen AK, Salminen HJ, Dean PB, De Crombrugghe B, Vuorio EI, et al. (2000) Osteoarthritis-like lesions in transgenic mice harboring a small deletion mutation in type II collagen gene. Osteoarthritis Cartilage 8: 248-57.

40. Neuhold LA, Killar L, Zhao W, Sung ML, Warner L, et al. (2001) Postnatal expression in hyaline cartilage of constitutively active human collagenase-3 (MMP13) induces osteoarthritis in mice. J Clin Invest 107: 35-44.

41. Hu K, Xu L, Cao L, Flahiff CM, Brussiau J (2006) Pathogenesis of osteoarthritis-like changes in the joints of mice deficient in type IX collagen. Arthritis Rheum 54: $2891-900$

42. Guingamp C, Gegout-Pottie P, Philippe L, Terlain B, Netter P, et al. (1997) Mono-iodoacetate-induced experimental osteoarthritis: a dose-response study of loss of mobility, morphology, and biochemistry. Arthritis Rheum 40: 1670-9.

43. Murat N, Karadam B, Ozkal S, Karatosun V, Gidener S (2007) Quantification of papain-induced rat osteoarthritis in relation to time with the Mankin score. Acta Orthop Traumatol Turc 41: 233-7.

44. Yeh TT, Wen ZH, Lee HS, Lee CH, Yang Z, et al. (2008) Intra-articular injection of collagenase induced experimental osteoarthritis of the lumbar facet joint in rats. Eur Spine J 17: 734-42. 
45. van der Kraan PM, Vitters EL, van de Putte LB, van den Berg WB (1989) Development of osteoarthritic lesions in mice by "metabolic" and "mechanical" alterations in the knee joints. Am J Pathol 135: 1001-14.

46. Glasson SS, Chambers MG, Van Den Berg WB, Little CB (2010) The OARSI histopathology initiative - recommendations for histological assessments of osteoarthritis in the mouse. Send to Osteoarthritis Cartilage 3: S17-23.

47. Hayashi D, Roemer FW, Guermazi A (2016) Imaging for osteoarthritis. Ann Phys Rehabil Med 59: 161-9.

48. Guermazi A, Hunter DJ, Li L, Benichou O, Eckstein F, et al. (2012) Different thresholds for detecting osteophytes and joint space narrowing exist between the site investigators and the centralized reader in a multicenter knee osteoarthritis study--data from the Osteoarthritis Initiative. Skeletal Radiol 41: $179-86$.

49. Dacre JE, Coppock JS, Herbert KE, Perrett D, Huskisson EC (1989) Development of a new radiographic scoring system using digital image analysis. Ann Rheum Dis 48: 194-200

50. Lynch JA, Buckland-Wright JC, Macfarlane DG (1993) Precision of joint space width measurement in knee osteoarthritis from digital image analysis of high definition macroradiographs. Osteoarthritis Cartilage 1: 209-18.

51. Duryea J, Neumann G, Niu J, Totterman S, Tamez J, et al. (2010) Comparison of radiographic joint space width with magnetic resonance imaging cartilage morphometry: analysis of longitudinal data from the Osteoarthritis Initiative. Arthritis Care Res 62: 932-7.

52. Burr DB, Schaffler MB (1997) The involvement of subchondral mineralized tissues in osteoarthrosis: quantitative microscopic evidence. Microsc Res Tech 37: 343-57.

53. Piscaer TM, van Osch GJ., Verhaar JA, Weinans H (2008) Imaging of experimental osteoarthritis in small animal models. Biorheol 45: 355-64.

54. Waarsing JH, Day JS, Weinans H (2005) Longitudinal micro-CT scans to evaluate bone architecture. J Musculoskelet Neuronal Interact. 5: 310-2.

55. Wachsmuth L, Engelke K (2004) High-resolution imaging of osteoarthritis using microcomputed tomography. Cartilage Osteoarthritis 101: 231-48.

56. Botter SM, van Osch GJ, Waarsing JH, Day JS, Verhaar JA, et al. (2006) Quantification of subchondral bone changes in a murine osteoarthritis model using micro-CT. Biorheology 43: 379-88.

57. McErlain DD, Appleton CT, Litchfield RB, Pitelka V, Henry JL, et al. (2008) Study of subchondral bone adaptations in a rodent surgical model of OA using in vivo micro-computed tomography. Osteoarthritis Cartilage 16: 458-69.

58. Botter SM, van Osch GJ, Clockaerts S, Waarsing JH, Weinans H, et al. (2011) Osteoarthritis induction leads to early and temporal subchondral plate porosity in the tibial plateau of mice: an in vivo microfocal computed tomography study. Arthritis Rheum 63: 2690-9.

59. Kotwal N, Li J, Sandy J, Plaas A, Sumner DR (2012) Initial application of EPIC-muCT to assess mouse articular cartilage morphology and composition: effects of aging and treadmill running. Osteoarthritis Cartilage 20: 887-95.

60. Ruan MZ, Dawson B, Jiang MM, Gannon F, Heggeness M, et al. (2013) Quantitative imaging of murine osteoarthritic cartilage by phase-contrast microcomputed tomography. Arthritis Rheum 65: 388-96.

61. Lakin BA, Patel H1, Holland C, Freedman JD, Shelofsky JS, et al. (2016) Contrast-enhanced CT using a cationic contrast agent enables non-destructive assessment of the biochemical and biomechanical properties of mouse tibial plateau cartilage. J Orthop Res 34: 1130-8.

62. Ostergaard M, Hansen M, Stoltenberg M, Lorenzen I (1996) Quantitative assessment of the synovial membrane in the rheumatoid wrist: an easily obtained MRI score reflects the synovial volume. Br J Rheumatol 35: 965-71.

63. Proulx ST, Kwok E, You Z, Papuga MO, Beck CA, et al. (2007) Longitudinal assessment of synovial, lymph node, and bone volumes in inflammatory arthritis in mice by in vivo magnetic resonance imaging and microfocal computed tomography. Arthritis Rheum 56: 4024-37.

64. Proulx ST, Kwok E, You Z, Beck CA, Shealy DJ, et al. (2007) MRI and quantification of draining lymph node function in inflammatory arthritis. Ann N Y Acad Sci 1117: 106-23.

65. Goebel JC, Pinzano A, Grenier D, Perrier AL, Henrionnet C, et al. (2010) New trends in MRI of cartilage: Advances and limitations in small animal studies. Biomed Mater Eng 20: 189-94.

66. Munasinghe JP, Tyler JA, Carpenter TA, Hall LD (1995) High resolution MR imaging of joint degeneration in the knee of the STR/ORT mouse. Magn Reson Imaging 13: 421-8.

67. Goebel JC, Bolbos R, Pham M, Galois L, Rengle A, et al. (2010) In vivo high-resolution MRI (7T) of femoro-tibial cartilage changes in the rat anterior cruciate ligament transection model of osteoarthritis: a cross-sectional study. Rheumatology 49: 1654-64.

68. Horger M, Bares R (2006) The role of single-photon emission computed tomography/computed tomography in benign and malignant bone disease. Semin Nucl Med 36: 286-94.

69. Lee E, Worsley DF (2006) Role of radionuclide imaging in the orthopedic patient. Orthop Clin North Am 37: 485-501.

70. Goupille P, Chevalier X, Valat JP, Garaud P, Perin F, et al. (1997) Macrophage targeting with 99mTc-labelled J001 for scintigraphic assessment of experimental osteoarthritis in the rabbit. Br J Rheumatol 36: 758-62.

71. Scharf S (2009) SPECT/CT imaging in general orthopedic practice. Semin Nucl Med 39: 293-307.

72. Franc BL, Goth S, MacKenzie J, Li X., Blecha J, et al. (2017) In Vivo PET Imaging of the Activated Immune Environment in a Small Animal Model of Inflammatory Arthritis. Mol imaging 16: 1536012117712638.

73. Seabra J, Sanches JM (2012) RF Ultrasound Estimation from B-Mode Images. Ultrasound Imaging pp 3-24.

74. van Holsbeeck M, Introcaso JH (1992) Musculoskeletal ultrasonography. Radiol Clin North Am 30: 907-25.

75. Grassi W, Cervini C (1998) Ultrasonography in rheumatology: an evolving technique. Ann Rheum Dis 57: 268-71.

76. Zayat AS, Freeston JE, Conaghan PG, Hensor EM, Emery P, et al. (2012) Does joint position affect US findings in inflammatory arthritis? Rheumatology 51: 921-5.

77. Torp-Pedersen S, Christensen R, Szkudlarek M, Ellegaard K, D’Agostino MA, et al. (2015) Power and color Doppler ultrasound settings for inflammatory flow: impact on scoring of disease activity in patients with rheumatoid arthritis. Arthritis Rheumatol 67: 386-95.

78. Backhaus M, Burmester GR, Gerber T, Grassi W, Machold KP, et al. (2001) Guidelines for musculoskeletal ultrasound in rheumatology. Ann Rheum Dis 60: 641-9.

79. Mandl P, Naredo E, Wakefield RJ, Conaghan PG, D’Agostino MA, et al. (2011) A systematic literature review analysis of ultrasound joint count and scoring systems to assess synovitis in rheumatoid arthritis according to the OMERACT filter. J Rheumatol 38: 2055-62. 
80. Aletaha D, Neogi T, Silman AJ, Funovits J, Felson DT (2010) 2010 Rheumatoid arthritis classification criteria: an American College of Rheumatology/European League Against Rheumatism collaborative initiative. Arthritis rheum 62: 2569-81.

81. Colebatch AN, Edwards CJ, Ostergaard M, van der Heijde D, et al. (2013) EULAR recommendations for the use of imaging of the joints in the clinical management of rheumatoid arthritis. Ann Rheum Dis 72: 804-14.

82. Plaza M, Nowakowska-Plaza A, Pracon G, Sudol-Szopinska I (2016) Role of ultrasonography in the diagnosis of rheumatic diseases in light of ACR/EULAR guidelines. J Ultrason 16: 55-64.

83. D’Agostino MA, Terslev L, Wakefield R, Ostergaard M, Balint P, et al. (2016) Novel algorithms for the pragmatic use of ultrasound in the management of patients with rheumatoid arthritis: from diagnosis to remission. Ann Rheum Dis 75: 1902-08.

84. Riecke BF, Christensen R, Torp-Pedersen S, Boesen M, Gudbergsen H, et al. (2014) An ultrasound score for knee osteoarthritis: a cross-sectional validation study. Osteoarthritis Cartilage 22: 1675-91.

85. Hall M, Doherty S, Courtney P, Latief K, Zhang W, et al. (2014) Synovial pathology detected on ultrasound correlates with the severity of radiographic knee osteoarthritis more than with symptoms. Osteoarthritis Cartilage 22: 1627-33.

86. Birn J, Pruente R, Avram R, Eyler W, Mahan M, et al. (2014) Sonographic evaluation of hip joint effusion in osteoarthritis with correlation to radiographic findings. J Clin Ultrasound 42: 205-11.

87. Malas FU, Kara M, Kaymak B, Akinci A, Ozcakar L (2014) Ultrasonographic evaluation in symptomatic knee osteoarthritis: clinical and radiological correlation. Int J Rheum Dis 17: 536-40.

88. Abraham AM, Pearce MS, Mann KD, Francis RM, et al. (2014) Population prevalence of ultrasound features of osteoarthritis in the hand, knee and hip at age 63 years: the Newcastle thousand families birth cohort. BMC Musculoskelet Disord 15: 162.

89. Liukkonen J, Lehenkari P, Hirvasniemi J, Joukainen A, Viren T, et al. (2014) Ultrasound arthroscopy of human knee cartilage and subchondral bone in vivo. Ultrasound Med Biol 40: 2039-47.

90. Wang Y, Teichtahl AJ, Cicuttini FM (2016) Osteoarthritis year in review 2015: imaging. Osteoarthritis Cartilage 24: 49-57.

91. D'Agostino MA, Conaghan P, Le Bars M, Baron G, Grassi W, et al. (2005) EULAR report on the use of ultrasonography in painful knee osteoarthritis. Part 1: prevalence of inflammation in osteoarthritis. Ann Rheum Dis 64: 1703-9.

92. Conaghan P, D’Agostino MA, Ravaud P, Baron G, Le Bars M, et al. (2005) EULAR report on the use of ultrasonography in painful knee osteoarthritis. Part 2: exploring decision rules for clinical utility. Annals of the rheumatic diseases 64: 1710-4.

93. de Miguel Mendieta E, Cobo Ibáñez T, Usón Jaeger J, Bonilla Hernán G, Martín Mola E (2006) Clinical and ultrasonographic findings related to knee pain in osteoarthritis. Osteoarthritis Cartilage 14: 540-4.

94. Wakefield RJ, Balint PV, Szkudlarek M, Filippucci E, Backhaus M, et al. (2005) Musculoskeletal ultrasound including definitions for ultrasonographic pathology. J Rheumatol 32: 2485-7.

95. Keen, HI, Conaghan PG (2009) Ultrasonography in osteoarthritis. Radiol Clin North Am 47: 581-94.

96. Bruyn GA, Naredo E, Damjanov N, Bachta A, Baudoin P, et al. (2016) An OMERACT reliability exercise of inflammatory and structural abnormalities in patients with knee osteoarthritis using ultrasound assessment. Ann Rheum Dis 75: 842-6.

97. Spriet MP, Girard CA, Foster SF, Harasiewicz K, Holdsworth DW, et al. (2005) Validation of a $40 \mathrm{MHz}$ B-scan ultrasound biomicroscope for the evaluation of osteoarthritis lesions in an animal model. Osteoarthritis Cartilage 13: 171-9.

98. Hulth A, Lindberg L, Telhag H (1970) Experimental osteoarthritis in rabbits. Preliminary report. Acta Orthop Scand 41: 522-30.

99. Wang LV (2009) Multiscale photoacoustic microscopy and computed tomography. Nat Photonics 3: 503-9.

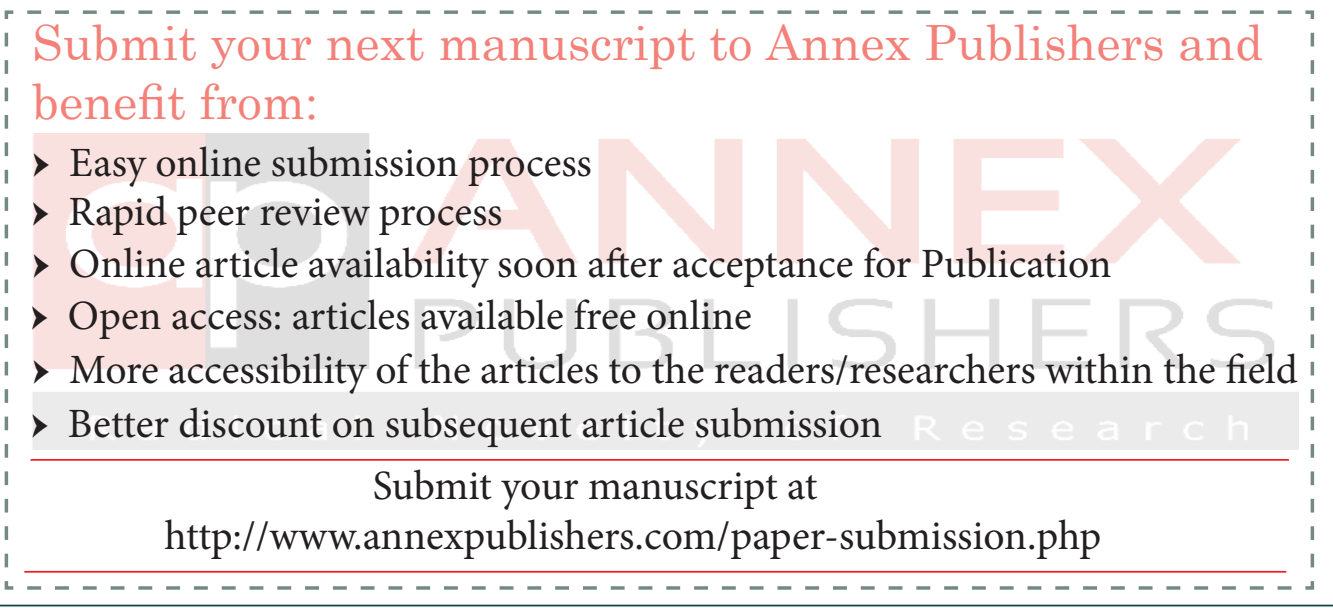

